\title{
Dynamics of Mesoscopic Precipitate Lattices in Phase Separating Alloys under External Load
}

\author{
R. Weinkamer ${ }^{1,2,3}$, H. Gupta ${ }^{2,3}$, P. Fratzl ${ }^{3}$, and J.L. Lebowitz ${ }^{2}$ \\ ${ }^{1}$ Institut für Materialphysik der Universität Wien, Strudlhofgasse 4, A-1090 Wien, Austria \\ ${ }^{2}$ Departments of Mathematics and Physics, Rutgers University, Busch Campus, New Brunswick, 08903 New Jersey, USA \\ ${ }^{3}$ Erich Schmid Institut für Materialwissenschaft, Österreichische Akademie der Wissenschaften 8 Institut für Metallphysik, \\ Montanuniversität Leoben, Jahnstraße 12, A-8700 Leoben, Austria
}

\begin{abstract}
We investigate, via three-dimensional atomistic computer simulations, phase separation in an alloy under external load. A regular two-dimensional array of cylindrical precipitates, forming a mesoscopic precipitate lattice, evolves in the case of applied tensile stress by the movement of mesoscopic lattice defects. A striking similarity to ordinary crystals is found in the movement of "meso-dislocations", but new mechanisms are also observed. Point defects such as "meso-vacancies" or "meso-interstitials" are created or annihilated locally by merging and splitting of precipitates. When the system is subjected to compressive stress, we observe stacking faults in the mesoscopic one-dimensional array of plate-like precipitates.
\end{abstract}

The coarsening of precipitates in a phase separating alloy usually proceeds via an Ostwald ripening process where large particles grow at the expense of smaller ones [1]. The driving force for this process is the tendency to reduce the total amount of interface between matrix and precipitates. The coarsening kinetics follows the laws of dynamical scaling, with a characteristic length $R(t)$ growing like $t^{1 / 3}$, as first described by the theory of Lifshitz, Slyozov and Wagner (LSW theory) when the density of precipitate particles is small [2,3]. The situation changes radically when a difference in lattice spacing between matrix and precipitates (lattice misfit) gives rise to long-range elastic interactions. The precipitates then tend to be cuboidal or plate-like instead of spherical and start to align themselves into more or less regular arrays to minimize the elastic energy [4 [8]. Changes in the growth kinetics of precipitates, especially a slowing-down in the later stages, have also been reported [9,10]. When a uniaxial external stress is applied, the cubic symmetry is broken and precipitates arrange themselves into arrays of cylinders parallel to or of plates perpendicular to the direction of external stress (rafting) [11 16.

During the coarsening process in an alloy with lattice misfit, the number of precipitates reduces, implying an increase in the spacing between them. Since this cannot occur continuously in a regular array of precipitates, Wang et al. [17 have proposed defects within the mesoscopic lattice of precipitates to allow the necessary rearrangement in a way very similar to dislocation climbs in ordinary crystals. Nothing is known, however, about the detailed mechanisms that allow the generation and the movement of such mesoscopic defects in order to enable the coarsening process. The dynamics of these defects rather than the atomic diffusion alone, as in the LSW theory, will have a decisive effect on the growth kinetics of the precipitates. Similar phenomena may be expected in the coarsening of the two-dimensional arrays of cylinders or the one-dimensional array of plates occurring as a consequence of rafting under uniaxial stress.

To clarify the mechanisms by which the precipitate lattices coarsen, it is necessary to investigate the details of the local arrangements of the precipitates and their movement, in the same way as it is necessary to study local atomic arrangements for the detailed investigation of dislocation movements at the atomic level. While the latter is possible with transmission electron microscopy, the first is much more difficult if not impossible with direct imaging or scattering techniques. In the present paper, we study this phenomenon by three-dimensional computer simulations using an atomistic lattice model that has proven effective in the simulation of phase separation with lattice misfit in two dimensions [18,12]. One of the advantages of such computer simulations is that the process can be followed "in-situ" by monitoring the evolution of individual precipitates. An investigation of phase separation under external load has the further advantage that the dimensionality of the precipitate lattices is lowered due to the external stress (two-dimensional for tensile, one-dimensional for compressive stress, respectively) making an analysis of the coarsening process easier. In the case of cylindrical precipitates parallel to the direction of tensile stress, it turns out that the "meso-lattice" formed by the projected cylinder axes evolves by the movement of lattice defects. We want to emphasize the similarity of their appearance to atomistic lattice defects by naming them "meso-dislocations", "meso-vacancies" and "meso-interstitials". Whereas meso-dislocations move in a way very similar to dislocations in an ordinary crystal, the dynamics of meso-vacancies and -interstitials is totally different from their analog in atomic lattices. There is no long-range diffusion of these defects, but they are created or annihilated locally by splitting or merging of cylindrical precipitates. In the case of an applied compressive stress, we show the existence of "meso-stacking faults" in the linear array of plates perpendicular to the direction of stress.

We use a conceptually simple three-dimensional atomistic model for a binary alloy containing only parameters which are experimentally accessible. A face-centered cubic (fcc) lattice $\mathcal{L}$ with $N$ lattice sites and periodic boundary conditions is occupied by two types of atoms, $A$ and $B$. A spin variable $\gamma(\mathbf{p})$ is assigned at each $\mathbf{p} \epsilon \mathcal{L}$, with $\gamma(\mathbf{p})=1$ 
if there is an $A$ atom at site $\mathbf{p}$ and $\gamma(\mathbf{p})=-1$ if there is a $B$ atom at this site. A chemical interaction between nearest-neighbor atoms is assumed with like atoms attracting one another. To model the elastic interactions caused by the different sizes of $A$ and $B$ atoms, all nearest-neighbor pairs of atoms are considered to be connected by springs with a longitudinal and two different transverse spring constants. The Hamiltonian of this microelastic model can be written as a quadratic form of the spin variables $\gamma(\mathbf{p})$ or, using their Fourier transforms $\tilde{\gamma}(\mathbf{k})$, as [19, 20, 5, 18, 12,

$$
\mathcal{H}=\frac{1}{2 N} \sum_{\mathbf{k}} \boldsymbol{\Psi}(\mathbf{k})|\tilde{\gamma}(\mathbf{k})|^{2}
$$

when it is assumed that the spring constants are independent of composition and the relaxation time of the lattice distortions is much shorter than the diffusion time of the atoms. In the case of external applied stresses and only a weak dependence of the spring constants on the type of atoms they connect, the expression (1) remains approximately valid but the interaction potential $\mathbf{\Psi}(\mathbf{k})$ does not have same symmetry as the crystal lattice (for details on the model see [12]).

The atomic configurations on the lattice evolve by the Metropolis algorithm with Kawasaki exchange dynamics of nearest-neighbor atoms 21]. To deal with the long-range interactions a special updating procedure was employed [18]. The time unit is one Monte Carlo step (MCS), i.e. one attempted exchange per site. We carried out simulations on a lattice $\mathcal{L}$ with 48 cubic cells in each direction, i.e. 442368 lattice sites. The size difference between A and B atoms was taken to be $1 \%$. The spring constants in the model correspond to Born-von Karman parameters, which can be obtained directly from the measured phonon dispersion of the crystal [22]. Choosing the parameters of copper, our model resembles elastically a typical metal with negative elastic anisotropy. The concentration of A atoms was taken $\bar{c}=0.2$. Starting from a homogeneous configuration, the time evolution of the system was monitored after a quench to a temperature within the two-phase region $T / T_{c} \approx 0.4$, where $T_{c}$ denotes the critical temperature for phase separation determined approximately by a series of MC runs. Finally, the sign of the misfit was chosen in such a way that cylindrical precipitates form under tensile stress and plates under compressive stress [11,12].

In Figure 1 the mean precipitate size $R(t)$ is plotted as a function of $t^{1 / 3} . R(t)$ is defined as $R(t) \equiv \frac{2 \bar{c}}{P_{A B}}$, where $P_{A B}$ is the probability of a nearest-neighbor $A B$ atom-pair. This definition assumes that during coarsening $R(t)$ is inversely proportional to the interface between precipitate and matrix, which is estimated in our model by the probability $P_{A B}$. Besides the symbols representing the results for the cases of tensile, compressive and no external stress, the solid line depicts $R(t)$ for an $f c c$ Ising model with only nearest neighbor chemical interactions at the same temperature compared to $T_{c}$. After $600 \mathrm{MCS}$ the growth of the precipitates slows down substantially from the usual power law $R(t) \propto t^{1 / 3}$ in the case when elastic interactions are considered, although $R(t)$ is nearly insensitive to the sign of the external load. An influence on $R(t)$ due to the finite system size cannot be fully excluded. A study of a system with comparable system size (but with short range chemical interactions only) did not show any finite-size effects at the comparatively early times $(t<6000 \mathrm{MCS})$ we consider here [23].

To understand the processes leading to this slowing down, we looked in detail at the time evolution of the precipitate microstructure. Figure 2 shows the configuration obtained after 3000 MCS when the system was subjected to tensile stress. To reduce the three-dimensional configuration to a two-dimensional plot, we averaged the concentration of $A$ atoms along lines normal to the plotting plane. Therefore the plot can be read in a way similar to an X-ray transmission micrograph. An analysis of the full three-dimensional data as well as the structure function confirmed that the more or less regularly arranged bright spots in Fig. 2 correspond to $A$-rich, cylindrical precipitates with their axis parallel to the stress direction. For a more detailed analysis of the kinetics, we have redrawn these "micrographs" using contour lines to indicate the amount of $A$ atoms along a line perpendicular to the plot (Fig. 3). For a clearer representation of the data, they were smoothed by averaging the value of each pixel with all of its first neighbors. Peaks in the amount of $A$ atoms are visible in the contour plots and can be interpreted as points of a "meso-lattice" of parallel cylindrical precipitates. These points were connected by lines revealing a distorted square lattice reflecting the symmetry of the elastically soft $\langle 100\rangle$-directions. Contour lines were plotted for steps of 0.1 in the averaged concentration of $A$ atoms starting at $c=\bar{c}=0.2$. The highest values around the "meso-lattice points" correspond to $c \geq 0.9$. In some cases, however, the height is significantly smaller (e.g., the point just below the number 4 in Fig. 3a). These correspond to cylinders which do not extend over the full thickness of the specimen along the line of projection. Tilted or kinked cylinders may give elongated spots in the contour line plot (e.g., close to number 8 and 11 in Fig. 3c).

The number of cylindrical precipitates decreases from 72 at $600 \mathrm{MCS}$ to 49 at $3000 \mathrm{MCS}$. The spacing of the mesoscopic lattice increases visibly during this period. A measure for this increase is the number of rows and columns as given by the square root of the number of precipitates. Starting with 8.48 (600 MCS), the number decreases continuously to 8.19 (1200 MCS), 7.35 (2000 MCS) and finally 7 (3000 MCS). Such a continuous change via non- 
integer numbers is, however, impossible in a perfect square lattice. Indeed, Fig. 3 shows defects responsible for this increase in the meso-lattice parameter via the following dynamical processes:

1) Dynamics of meso-interstitials: At 600 MCS we observe a number of precipitates which do not occupy regular lattice sites (marked by open circles in Fig. 3). We call these meso-interstitials. The contours show that mesointerstitials dissolve by a flow of $A$ atoms along elastically soft directions towards a nearest-neighbor precipitate on a regular lattice site. In some cases this also leads to a shift of the defect which finally merges with the precipitate of the meso-lattice site (arrow in Fig. 3a,b). Most of these meso-interstitials are not fully developed cylinders as indicated by their relatively low content in $A$ atoms as compared to regular lattice sites. During the period covered by Fig. 3, new meso-interstitials (Fig. 3c) are not formed by precipitate nucleation, but by rearrangement of the meso-lattice (see point 3 below).

2) Dynamics of meso-vacancies: Splitting of precipitate cylinders is also possible (arrow in Fig. 3d). In the case shown, a precipitate splits to fill a neighboring meso-vacancy (number $11 \mathrm{in} \mathrm{Fig.} \mathrm{3c).} \mathrm{Meso-vacancies} \mathrm{are} \mathrm{represented}$ by squares in Fig. 3. They typically are formed by dissolution of precipitates on regular meso-lattice sites, which is one of the mechanisms to reduce the total number of lattice points (see vacancies 9 to 11 in Fig. 3c,d).

3) Dynamics of meso-dislocations: In addition to the already mentioned vacancies and interstitials, the meso-lattice also shows the analog of dislocations (denoted by the symbol $\perp$ ). They interact with point defects and also with each other to increase the spacing and regularity of the meso-lattice. Examples for such complex reactions are visible in Fig. 3. First, the column of points bounded by $\perp 1$ and $\perp 4$ is gradually disappearing from Fig. 3a to 3c by dissolution of precipitates leading to the annihilation of the meso-dislocations. The start of a similar process can be seen with the formation of vacancy 9 and the subsequent dissolution of the two lattice points immediately below (which has almost led to their disappearance in Fig. 3d). Another process starts with the dissolution of a single lattice point (denoted by $\star$ in Fig. 3a). The resulting meso-vacancy splits into two dislocations (denoted $\perp 7$ and $\perp 8$ in Fig. 3b). $\perp 7$ is attracted by $\perp 3$ and they finally annihilate leaving behind two interstitials (Fig. 3c) which both dissolve. Only the remains of one of these two are still visible in Fig. 3d.

When the system is subjected to compressive stress, our simulations show a one-dimensional array of plate-like precipitates corresponding to regions of high concentration of A atoms perpendicular to the direction of applied stress. These plate like precipitates are separated by regions with hardly any A atoms at all. Fig. 4 shows on top the one-dimensional concentration profile of $A$ atoms along the stress direction. Near the middle of the system there are large defects almost washing out the periodic structure. But even where the plates seem well-defined, lattice defects can be observed. This is illustrated by the three pictures at the bottom which give the distribution of $A$ atoms within planes perpendicular to the direction of stress. Holes in the plate-like precipitates (plate 1 and plate 2 in Fig. 4) are aligned along the elastically soft cubic directions. The most striking feature, however, is the defect highlighted by the dashed rectangle in all three pictures. In this region, the plates have a large hole, while in the gap between the plates (gap in Fig. 4) an $A$-rich area can be seen. This highlighted region with inverted content of $A$-atoms therefore corresponds to a stacking fault of the meso-lattice. A schematic view of the defect (with the components plate 1 , gap and plate 2) is also shown in Fig. 4. Such stacking faults occur frequently and are extremely long-lived in the time-evolution of the alloy, particularly at low temperatures.

The occurrence of defects in mesoscopic precipitate lattices with totally different topology suggests that they are a common feature when coherent elastic strains are important in the phase transformation process. We expect that similar defects can be found in a system, which is not subjected to an additional external stress. The simplified topology due to the external stress helped to identify several types of defects, showing similarities and some striking differences with respect to defects in ordinary crystal lattices. In contrast to the usual Ostwald ripening process, coarsening is mediated by the movement of these mesoscopic defects, which allows a reduction of the number of precipitates without destroying the mesoscopic lattice structure.

\section{ACKNOWLEDGMENTS}

The work of H.G. and J.L.L. was supported by NSF Grant No. NSF-DMR-9813268. 
[1] R. Wagner and R. Kampmann, in Materials Science and Technology, edited by P. Haasen (VCH Verlagsges., Weinheim, 1991), Vol. 5, Chap. 4, p. 213; K. Binder, ibid., Chap. 7, p. 405.

[2] I.M. Lifshitz and V.V. Slyozov, J. Phys. Chem. Solids 19, 35 (1961); C. Wagner, Z. Elektrochem. 65, 581 (1961).

[3] For a review see: J.D. Gunton, M. San Miguel and P.S. Sahni, in Phase Transitions and Critical Phenomena, edited by C. Domb and J.L. Lebowitz (Academic Press, New York, 1983), Vol. 8, p. 267; cf. J. Alkemper, V. A. Snyder, N. Akaiwa, and P. W. Voorhees, Phys. Rev. Lett. 82, 2725 (1999).

[4] For a recent review see: P. Fratzl, O. Penrose and J.L. Lebowitz, J. Stat. Phys. 95, 1429 (1999).

[5] A.G. Khachaturyan, Theory of Structural Transformations in Solids, (Wiley, New York, 1983).

[6] M.E. Thompson and P.W. Voorhees, Acta mater. 47, 983 (1999).

[7] C.H. Su and P.W. Voorhees, Acta mater. 44, 1987 (1996); ibid. 2001 (1996).

[8] D. Orlikowski, C. Sagui, A. Somoza and C. Roland, Phys. Rev. B 59, 8646 (1999).

[9] T. Miyazaki and M. Doi, Mater. Sci. Eng. A 110, 175 (1989).

[10] A. Onuki and H. Nishimori, Phys. Rev. B 43, 13649 (1991).

[11] J.C. Chang and S.M. Allen, J. Mater. Res. 6, 1843 (1991).

[12] C.A. Laberge, P. Fratzl and J.L. Lebowitz, Phys. Rev. Lett. 75, 4448, (1995); C.A. Laberge, P. Fratzl and J.L. Lebowitz, Acta mater. 45, 3949, (1997).

[13] W. Hort and W.C. Johnson, Met. and Mater. Trans. 27A, 1461 (1996).

[14] J.K. Lee, Mater. Sci. Eng. A 238, 1 (1997).

[15] D.Y. Li and L.Q. Chen, Scripta mater. 371271 (1997).

[16] D.Y. Li and L.Q. Chen, Acta mater. 47, 247 (1999).

[17] Y. Wang, L.Q. Chen and A.G. Khachaturyan, Acta metall. mater. 41, 279 (1993).

[18] P. Fratzl and O. Penrose, Acta metall. mater. 43, 2921, (1995); P. Fratzl and O. Penrose, Acta mater. 44, 3227 (1996).

[19] A.G. Khachaturyan, Soviet Phys. Crystallogr. 10, 248 (1965).

[20] H.E. Cook and D. DeFontaine, Acta metall. 17, 915 (1969).

[21] K. Binder and D.W. Heermann, Monte Carlo Simulation in Statistical Physics - An Introduction, (Springer, Berlin, 1988).

[22] P.H. Dederichs and H. Schober, in Landolt-Börnstein III/13a, (Springer, Berlin, 1981).

[23] D.W. Heermann, L. Yixue and K. Binder, Physica A 230132 (1996).

FIG. 1. Time evolution of the mean precipitate size $R(t)$ on a $t^{1 / 3}$ scale for the case of applied tensile (squares), compressive (circles) and no external stress (triangles) at a temperature $T / T_{c} \approx 0.4$. The solid line corresponds to an Ising model with nearest neighbor chemical interactions only.

FIG. 2. Concentration of solute $A$ atoms after 3000 MCS averaged along the direction of tensile stress, i.e., normal to the plotting plane. Bright (dark) regions represent a high (low) concentration of A atoms.

FIG. 3. Time evolution of the two-dimensional array of cylindrical precipitates (black points) formed under tensile stress: a) $600 \mathrm{MCS}$, b) $1200 \mathrm{MCS}$, c) $2000 \mathrm{MCS}$ and d) 3000 MCS. Lattice defects in the mesoscopic precipitate lattice, such as meso-dislocations (denoted by $\perp$ ), meso-vacancies $(\square)$ and meso-interstitials $(\bigcirc)$ are marked and labeled. The underlying contour plots provide information about the concentration of $A$ atoms averaged along the direction of the tensile stress.

FIG. 4. One-dimensional concentration profile of $A$ atoms (graph at the top) along the direction of compressive stress after 3000 MCS. The three pictures at the bottom show the concentration of $A$ atoms normal to the direction of stress after averaging over several neighboring lattice planes; a total of 6 lattice planes for the plate-like precipitates (plates 1 and 2 ) and 8 lattice planes for the gap in-between. Regions of high $A$ atom concentration are bright. The dashed line highlights a mesoscopic stacking fault in the one-dimensional array of plate-like precipitates. A three dimensional schematic view of the defect is given in the middle of the figure, where the arrows mark the direction of the external stress. 


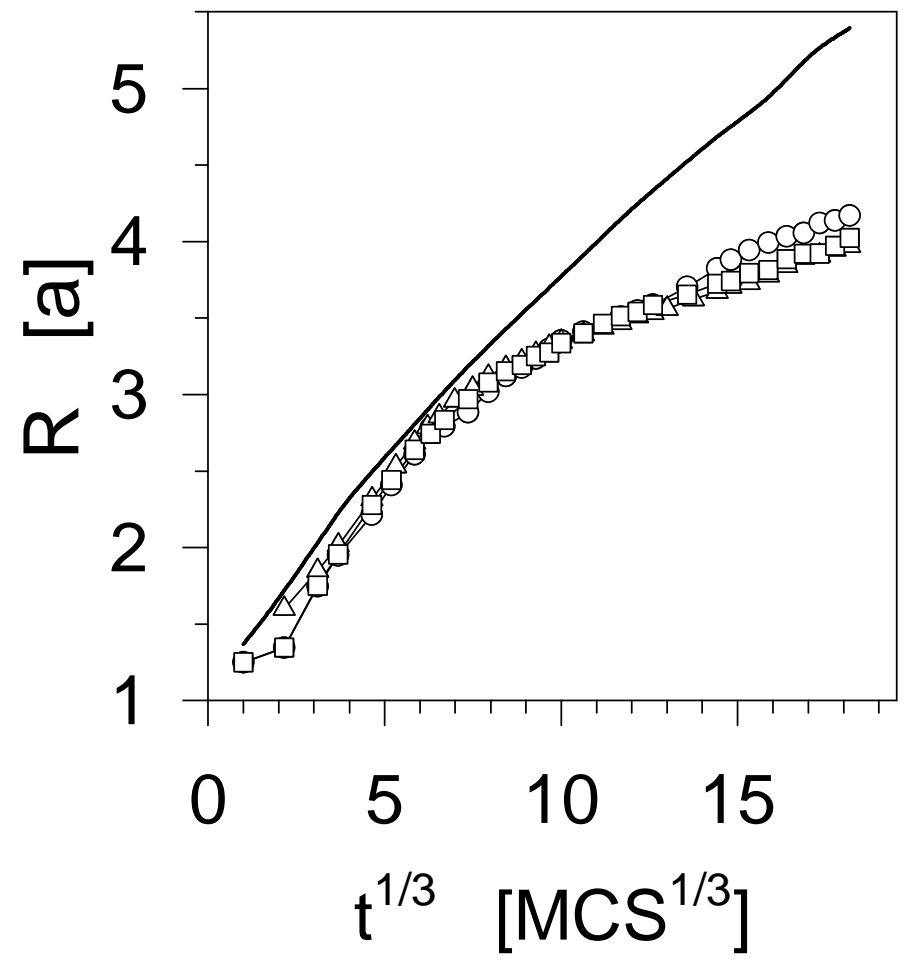




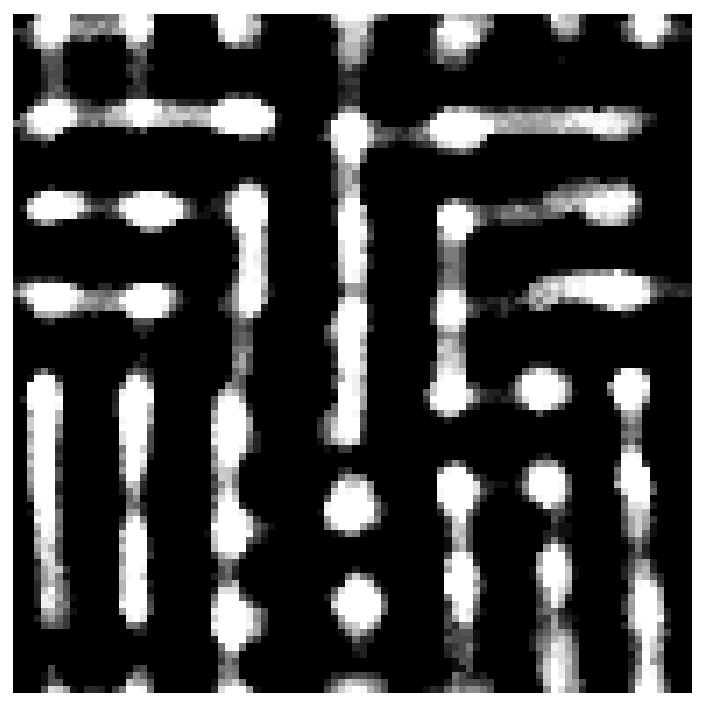



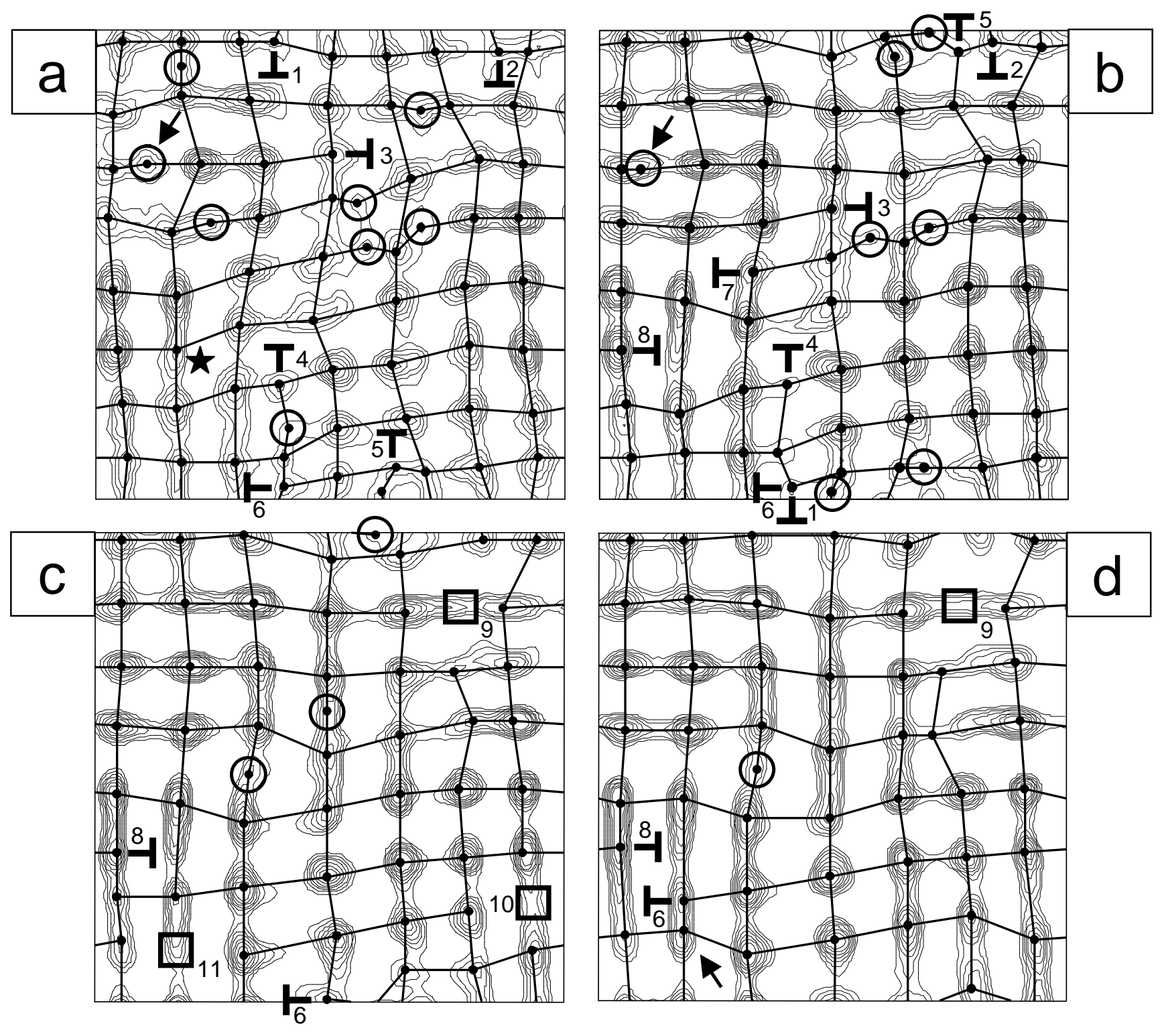


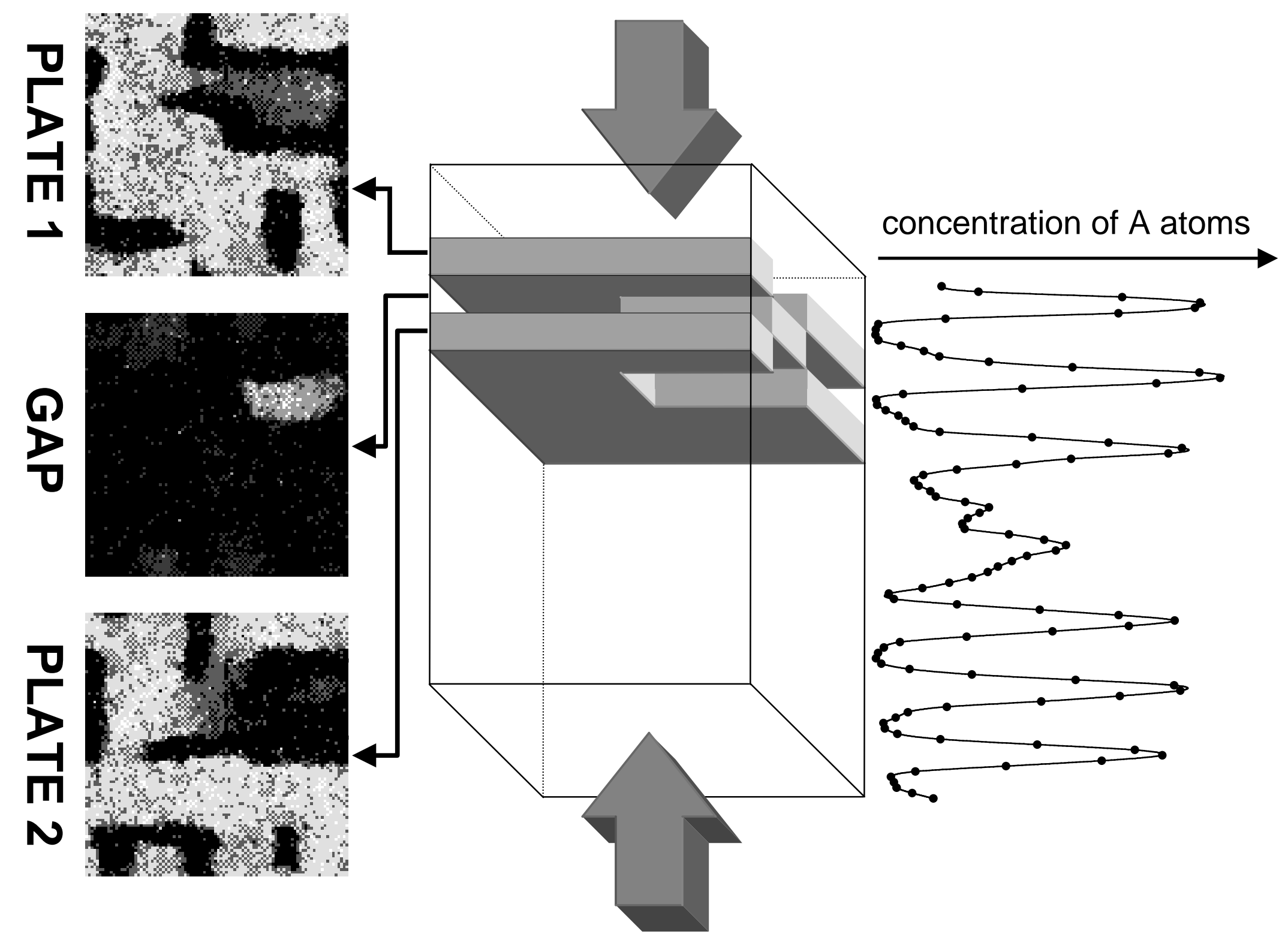

